2. Galvez-Olortegui JK, Álvarez-Vargas ML, Galvez-Olortegui TV, Godoy-Palomino A, Camacho-Saavedra L. Current clinical practice guidelines in atrial fibrillation: a review. Medwave. 2016;16:e6365.

3. Camm CF, Camm AJ. An explanation of recommendation differences: illustrations from recent atrial fibrillation guidelines. Pacing Clin Electrophysiol. 2014;37:116-27.

4. Weitz JI, Semchuk W, Turpie AGG, Fisher WD, Kong C, Ciaccia $\mathrm{A}$, et al. Trends in prescribing oral anticoagulants in Canada, 2008-2014. Clin Ther. 2015;37, 2506-14.e4.

5. Furie KL, Goldstein LB, Albers GW, Khatri P, Neyens R, Turakhia MP, et al. Oral antithrombotic agents for the prevention of stroke in nonvalvular atrial fibrillation: a science advisory for healthcare professionals from the American Heart Association/American Stroke Association. Stroke. 2012:3442-53.

6. Meschia JF, Bushnell C, Boden-Albala B, Braun LT, Bravata DM, Chaturvedi $\mathrm{S}$, et al. Guidelines for the primary prevention of stroke: A statement for healthcare professionals from the American heart association/American stroke association. Stroke. 2014, 3754-832 p.

7. Pinto DA, Sánchez-Vallejo CA, López Pedraza A, Vergara EP, Sáenz ÓA, González F, et al. Descripción de los pacientes con fibrilación auricular no valvular que ingresan al servicio de urgencias. Rev Colomb Cardiol. En prensa 2015.

8. Atun R, de Andrade LOM, Almeida G, Cotlear D, Dmytraczenko T, Frenz P, et al. Health-system reform and universal health coverage in Latin America. Lancet. 2015;385:1230-47.

9. Campillo-Artero C, Ortún V. El análisis de coste-efectividad: por qué y cómo. Rev Esp Cardiol. 2016;69:370-3.

10. Sanabria C, Cabrejos J, Olortegui A, Guevara C, Garrido Lecca S. Costo-efectividad de apixaban con otros noacs (dabigatran y rivaroxaban) en el tratamiento de la fibrilacion auricular no valvular (FANV) en pacientes de la Seguridad Social de Perú. Value Health. 2015; 18:A829.

11. Fernández Ávila Y, Garcia KC, Garrido Lecca S, Donato BM, Juarez-Garcia A. Cost-effectiveness of apixaban versus other new oral anticoagulants and warfarin for stroke prevention in atrial fibrillation in Venezuela. Value Health. 2015; 18:A829.

12. Tanaka S, Preto MC, Bernardino G, Nogueira F, Ferreira CN, Donato BM. Cost-effectiveness of apixaban versus other noacs and warfarin, during hospitalization in the private brazilian health system. Value Health. 2015;18:A830.

13. Giorgi MA, Caroli C, Giglio ND, Micone P, Aiello E, Vulcano C, et al. Estimation of the cost-effectiveness of apixaban versus vitamin $\mathrm{K}$ antagonists in the management of atrial fibrillation in Argentina. Health Econ Rev. 2015;5:52.
14. Triana JJ, Castañeda C, Parada L, Otálora-Esteban M, Rosselli D. Costo-efectividad de dabigatrán comparado con warfarina para el tratamiento de pacientes con fibrilación auricular no valvular. En: Rev Colomb Cardiol. En prensa; 2015.

15. Peñaherrera E, Tettamanti D, Aguirre F, Puyol LF, Cárdenas E, Torres G, et al. Análisis de costo-efectividad de la warfarina vs. dabigatrán etexilato en fibrilación auricular en ecuador. Value Health. 2015;18:A830.

16. Garcia Peña AA. Costo-efectividad de los nuevos anticoagulantes orales en pacientes con fibrilacion auricular no valvular en Colombia. Value Health. 2015;18:A829-30.

17. Romero M, Alfonso Quiñones PA, Acero G, Marrugo R. Evaluación de costo-efectividad y costo-utilidad del uso de rivaroxabán en pacientes con fibrilación auricular no valvular frente a warfarina en el contexto ecuatoriano. Value Health. 2015;18:A806.

18. Instituto de Evaluación Tecnológica en Salud - IETS. Análisis de costo-efectividad de dabigatran, rivaroxaban y apixaban comparado con warfarina para prevención de fenómenos tromboembólicos e isquémicos en pacientes adultos con fibrilación auricular no valvular en Colombia. 2015.

Camilo Peña-Quispe ${ }^{a, b, c, *}$, Anghelo Velasquez-Ojeda ${ }^{a, d}$, Carlos Plasencia-Meza ${ }^{\mathrm{a}, \mathrm{c}, \mathrm{d}}$ y Jose Galvez-Olortegui ${ }^{\mathrm{c}, \mathrm{e}}$

${ }^{a}$ Escuela de Postgrado Universidad Privada Antenor Orrego, Trujillo, Perú

b Servicio de Medicina Interna, Hospital Regional Docente de Trujillo, Trujillo, Perú

c Scientia Clinical and Epidemiological Research Institute, Trujillo, Perú

'Departamento de Emergencia, Hospital Regional Docente de Trujillo, Trujillo, Perú

e Facultad de Medicina, Universidad Nacional de Trujillo, Trujillo, Perú

* Autor para correspondencia.

Correos electrónicos: Campeq02@hotmail.com, cpenaq@scientiaceri.com (C. Peña-Quispe).

Disponible en Internet el 21 de septiembre de 2016

http://dx.doi.org/10.1016/j.rccar.2016.06.005

0120-5633/

(c) 2016 Sociedad Colombiana de Cardiología y Cirugía

Cardiovascular. Publicado por Elsevier España, S.L.U. Este es un artículo Open Access bajo la licencia CC BY-NC-ND (http:// creativecommons.org/licenses/by-nc-nd/4.0/).

\section{Síndrome de Kounis, ¿el tipo de infarto olvidado?}

\section{Kounis syndrome, the forgotten type of infarction?}

\section{Sr. Editor:}

El síndrome de Kounis es una entidad infrecuente y potencialmente infradiagnosticada en la práctica clínica. Se considera relevante el conocimiento de esta patología debido a la necesidad de pensar en ella ante pacientes que desarrollan un síndrome coronario agudo en el contexto de una reacción anafiláctica, optimizando así el tratamiento en situaciones de riesgo vital. Se presenta el caso de un paciente con infarto agudo de miocardio diagnosticado como síndrome de Kounis.

Un varón de 59 años, con antecedentes de dislipemia, diabetes mellitus tipo 2 y tabaquismo, acudió al Servicio de Urgencias por un episodio de dolor torácico de características anginosas dos horas después de la ingesta de huevas de pescado. Presentaba una TA: $95 / 55 \mathrm{~mm} \mathrm{Hg}$ y una FC: $120 \mathrm{lpm}$. A la exploración física destacaba múltiples lesiones habonosas y pruriginosas generalizadas, siendo la auscultación cardio-pulmonar anodina. Se realizó un electrocardiograma que mostró elevación del segmento ST anterior. En el análisis de sangre se objetivó leucocitosis con desviación izquierda, así como eosinofilia, además de elevación de marcadores de necrosis cardiaca (CK $692 \mathrm{U} / \mathrm{l}$, 
Tn I $19 \mathrm{ng} / \mathrm{ml}$ ). Se realizó un ecocardiograma transtorácico observándose hipocinesia anteroapical así como fracción de eyección del ventrículo izquierdo moderadamente disminuida (FEVI 40\%). Se solicitó coronariografía urgente que objetivó una estenosis del $80 \%$ en descendente anterior media (DAm), así como lesiones no significativas en primera oblicua marginal y coronaria derecha. Se realizó angioplastia coronaria transluminal percutánea a DAm con implante de stent farmacoactivo. Además del tratamiento específico para cardiopatía isquémica, se optimizó su tratamiento con prednisona y dexclorfeniramina. La IgE específica en plasma y el prick test cutáneo resultaron positivos para anisakis, permitiendo establecer el diagnóstico de síndrome de Kounis.

El síndrome de Kounis, angina alérgica o infarto miocárdico alérgico, ha sido descrito como la aparición de síndrome coronario agudo en el contexto de una reacción alérgica ${ }^{1,2}$. En cuanto a su fisiopatología, el contacto del alérgeno con el organismo produce la activación y degranulación de los mastocitos, los cuales liberan mediadores inflamatorios. Por un lado, la histamina y los leucotrienos son vasoconstrictores coronarios; por otro lado, la triptasa y la quimasa activan las metaloproteinasas que son las responsables de la degradación del colágeno con la consiguiente erosión o rotura de la eventual placa de ateroma ${ }^{3}$.

Algunos alérgenos se han asociado a la aparición de síndrome de Kounis, fundamentalmente fármacos ${ }^{4}$ y picaduras de himenópteros, siendo excepcional su aparición tras la ingesta de pescado, como lo refleja este caso.

Se han descrito tres tipos de síndrome de Kounis: tipo I, en aquellos pacientes que no presentan factores de riesgo cardiovascular (FRCV) y tienen arterias coronarias normales; tipo II, aquellos con FRCV en quienes se documenta ateromatosis (como en el caso descrito); y tipo III, en los casos de trombosis intra-stent coronario. En cuanto al manejo de esta entidad, en el tipo I el tratamiento del evento alérgico puede ser suficiente para lograr revertir los síntomas; en los tipos II y III, además de tratar la enfermedad coronaria se recomienda el uso de corticoterapia y antihistamínicos, así como evitar los alérgenos causantes del cuadro ${ }^{5}$.
En conclusión, el síndrome de Kounis en una enfermedad muy poco frecuente, pero que debe tenerse en cuenta en pacientes que se presentan con un síndrome coronario agudo en el contexto de una reacción anafiláctica.

\section{Conflicto de intereses}

Los autores declaran no tener ningún conflicto de intereses.

\section{Bibliografía}

1. Kounis NG. Coronary hypersensitivity disorder: the Kounis syndrome. Clin Ther. 2013;35:563-71.

2. Vivas D, Rubira JC, Ortiz AF, Macaya C. Coronary spasm and hypersensitivity to amoxicillin: Kounis or not Kounis syndrome? Int J Cardiol. 2008;128:279-81.

3. Kounis NG. Kounis syndrome (allergic angina and allergic myocardial infarction): a natural paradigm? Int J Cardiol. 2006;110:7-14.

4. Gázquez V, Dalmau G, Gaig P, Gómez C, Navarro S, Mercé J. Kounis syndrome: report of 5 cases. J Investig Allergol Clin Immunol. 2010;20:162-5.

5. Rico Cepeda P, Palencia Herrejón E, Rodríguez Aguirregabiria MM. Síndrome de Kounis. Med Intensiva. 2012;36:358-64.

Patricia Lozano-Cruz ${ }^{a, *}$, David Vivas ${ }^{\mathrm{b}}$, Fernando Gutiérrez-Marcos ${ }^{a}$ y Julio De Miguel $^{a}$

a Servicio de Medicina Interna, Hospital Universitario Príncipe de Asturias, Alcalá de Henares, Madrid, España

b Instituto Cardiovascular, Hospital Clínico San Carlos, Madrid, España

* Autor para correspondencia. Correo electrónico: patrimed@gmail.com (P. Lozano-Cruz).

http://dx.doi.org/10.1016/j.rccar.2016.05.007 0120-5633/

(c) 2016 Sociedad Colombiana de Cardiología y Cirugía Cardiovascular. Publicado por Elsevier España, S.L.U. Este es un artículo Open Access bajo la licencia CC BY-NC-ND (http://creativecommons.org/licenses/by-nc-nd/4.0/). 